\title{
ÉTIENNE LE ROY. POURQUOI ET COMMENT LA JURIDICITÉ DES COMMUNS S'EST-ELLE IMPOSÉE DANS NOS TRAVAUX FONCIERS ?, RÉCIT D'UNE INITIATION. REGARDS SUR LE FONCIER, N 8, COMITÉ TECHNIQUE « FONCIER \& DÉVELOPPEMENT »/ AFD/ MEAE, 2019.
}

\section{Sigrid Aubert, Philippe Karpe}

\section{Éditions de la Sorbonne | «Revue internationale des études du développement »}

$2020 / 3 N^{\circ} 243$ | pages 272 à 273

ISSN 2554-3415

ISBN 9791035105624

Article disponible en ligne à l'adresse :

https://www.cairn.info/revue-internationale-des-etudes-dudeveloppement-2020-3-page-272.htm

Distribution électronique Cairn.info pour Éditions de la Sorbonne.

(C) Éditions de la Sorbonne. Tous droits réservés pour tous pays.

La reproduction ou représentation de cet article, notamment par photocopie, n'est autorisée que dans les limites des conditions générales d'utilisation du site ou, le cas échéant, des conditions générales de la licence souscrite par votre établissement. Toute autre reproduction ou représentation, en tout ou partie, sous quelque forme et de quelque manière que ce soit, est interdite sauf accord préalable et écrit de l'éditeur, en dehors des cas prévus par la législation en vigueur en France. Il est précisé que son stockage dans une base de données est également interdit. 
Étienne Le Roy

\section{Pourquoi et comment la juridicité des communs s'est-elle imposée dans nos travaux fonciers? Récit d'une initiation}

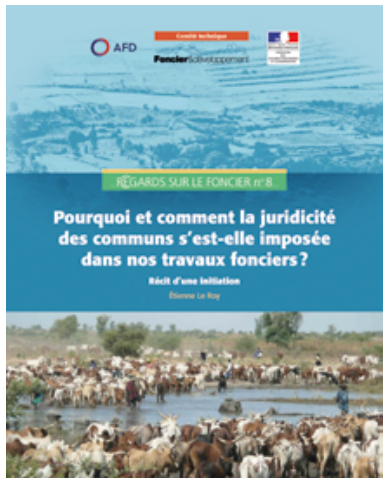

Regards sur le foncier, $\mathrm{n}^{\circ} 8$, Comité technique " Foncier \& développement "/ AFD/ MEAE, 2019.

L'auteur nous offre six décades de son parcours de "rebelle discipliné " (p. 15) auquel s'offrent les sociétés - africaines pour la plupart, mais pas seulement -, mais à laquelle les institutions résistent. Ce faisant, Étienne Le Roy nous conte I'histoire d'un droit qui s'émancipe sous son regard pour, in fine, offrir ses repères au commun et se mettre au service de ceux qui tissent des liens autour de la terre et de ses ressources. C'est bien d'une initiation dont il s'agit là, une invitation à emprunter le chemin de vie d'un chercheur, d'un juriste conscient et soucieux de ce qu'il est et doit être, un chemin semé d'embûches et de lumières que l'on suit avec une humilité et un enthousiasme sans cesse renouvelés par le mouvement des rencontres.

L'ouvrage d'une centaine de pages se présente sous la forme de trois parties. Les deux premières se succèdent chronologiquement pour restituer, au travers de l'histoire du développement des États, la manière dont l'auteur a élaboré sa recherche sur les rapports fonciers et, au-delà, de ce qu'ils expriment fondamentalement. La troisième partie rassemble des pensées alliées pour étoffer les fondations d'un cheminement à relayer et, ainsi, contribuer à promouvoir les communs jusqu'à révolutionner l'univers juridique de l'intérieur afin de restaurer la finalité essentielle du droit : il est proposé de retirer la gangue dans lequel le droit a été sciemment fossilisé.

Partant, dans les années 1960, d'un souci de " décolonisation juridique des territoires africains " (p. 11), les recherches entreprises au sein du tout jeune laboratoire d'anthropologie juridique de Paris ont été mises en dialogue avec celles investies par le monde anglo-saxon sur le pluralisme juridique. C'est un séjour en société wolof, au Sénégal, qui a révélé à Étienne Le Roy la complexité et la subtilité des rapports fonciers. Mais cette expérience le confronte cependant de plein fouet à la politique des États (le Sénégal et le Togo lui ferment leur frontière) et au dogmatisme des universités (I'agrégation d'histoire des institutions lui est refusée parce que I'Afrique et l'anthropologie ne figurent pas parmi les champs alors couverts par la discipline). II faudra une décennie pour que l'auteur puisse infiltrer ces places fortes et se faire entendre, tant en tant qu'expert des rapports fonciers qu'en tant qu'enseignant-chercheur. L'obtention d'un poste de professeur d'anthropologie du droit à I'université de Paris en 1987 va finalement permettre la constitution d'un socle solide afin de proposer une nouvelle approche " disciplinaire » : I'anthropologie politique de la juridicité.

Pour nous mettre en capacité d'observer l'existence et l'émergence d'une multitude de régimes de juridicités, l'auteur nous éclaire pour revenir sur les maîtrises 
foncières qu'il a théorisées dans le jeu des lois en 1999, non sans faire un détour dans les méandres de l'intermédiation culturelle, y compris en milieu judiciaire. En 1998, une méningite tente d'enrayer le flot créatif de son cerveau indiscipliné, mais les éléments du modèle se mettent peu à peu en place. L'ouvrage propose au fil des pages un lexique dont les termes sont précisément définis, illustrés et référencés, et invite aux échanges. On y apprend que la juridicité fait précéder les actes aux paroles sans s'attarder sur la logique monolithique du droit, tel qu'il est communément enseigné et publié. Elle restitue les paroles dans le sens de la pratique, dans le "faire commun", sous forme de transcriptions symboliques que les initiés confèrent aux mondes. Les éléments de pédagogie proposés par l'enseignant-chercheur deviennent alors des clés pour révéler des régimes de juridicité métis, combinés au gré du temps, des besoins et des milieux dans lesquels ils s'inscrivent. Le partage de ressources matérielles ou intellectuelles qualifié de rapport foncier s'impose comme un objet de recherche transculturel - et transdisciplinaire - qui se co-construit.

La troisième partie de l'ouvrage est une synthèse du patrimoine constitué par quelques autres illustres " juristes hétérodoxes " qui ont foulé le sentier de la juridicité des communs, parfois même sans le savoir, ajoutant une brique ou une curiosité à l'édifice. Car, pour parler de méthode, ces briques sont pour l'auteur autant de concepts, de paradigmes, de manières de dire et de faire qui nous permettent, au quotidien, de bousculer " nos modes d'observation et d'analyse des mutations en cours » (p. 75).

Cet ouvrage est le récit d'un passionné et d'un amoureux sincère de l'objet juridique qui communique avec humilité et courage sa volonté de lier, de relier, en accueillant les crispations et les blocages, les contraintes et l'ostracisme comme des promesses de découvertes. Si Étienne Le Roy s'est, durant de longues années, évertué à transmettre ses connaissances " aux rédacteurs des textes juridiques des pays anciennement colonisés » (p. 72), cet ultime ouvrage s'adresse aujourd'hui à ceux qui, ensemble, souhaitent mêler le " comprendre " et l' " agir " pour donner du sens et ouvrir de nouvelles places. "Pourquoi et comment la juridicité des communs s'est-elle imposée dans nos travaux fonciers? " est le partage de l'histoire d'une vie scientifique, d'une humanité mise à disposition des citoyens, étudiants, enseignants, chercheurs, praticiens et " passeurs " de multiples horizons...

Sigrid AUBERT

Philippe KARPE

CIRAD 